\title{
PRODUÇÃO DE SERAPILHEIRA EM FLORESTA DE GALERIA E FLORESTA MESOFÍTICA NA DOLINA DA GARAPA, DISTRITO FEDERAL, BRASIL
}

\author{
Otacílio Antunes Santana ${ }^{1}$, José Imaña Encinas² ${ }^{2}$ Flávio Luiz de Souza Silveira ${ }^{3}$, Gustavo Silva Ribeiro ${ }^{4}$
}

(recebido: 24 de abril de 2009; aceito: 30 de agosto de 2010)

\begin{abstract}
RESUMO: A serapilheira acumulada sobre o solo das florestas tem papel importante na dinâmica desses ecossistemas, pois a maior parcela da energia que flui no sistema está concentrada nesse compartimento. Objetivou-se, neste estudo, quantificar e analisar a serapilheira formada abaixo do dossel da Floresta de Galeria e Mesofítica, presentes na Dolina da Garapa-DF, relacionando-a com variáveis meteorológicas e ecofisiológicas, buscando verificar diferenças significativas entre estes dois ambientes florestais e entre os meses de estudo. De janeiro de 2006 a dezembro de 2007, foram dispostos 50 coletores de serapilheira em cada ambiente florestal, em que foi coletada, pesada e classificada a serapilheira em folhas, caule e galhos, flores, frutos e sementes. Os valores médios encontrados de produção de serapilheira em massa total de folhas, flores e caules, galhos, frutos e sementes, foram diferentes significativamente nos dois ambientes estudados. Na correlação entre as variáveis meteorológicas e ecofisiológias e o valor de serapilheira registrado, observou-se, que apenas a variável temperatura não obteve significância do coeficiente de correlação $(\rho<0,5)$ para produção de serapilheira. Pela produção total de serapilheira durante o período estudado mostrou-se que a Floresta Mesofítica é a mais vulnerável a fatores externos meteorológicos e a fatores ecofisiológicos de suas espécies, do que a Floresta de Galeria. Primeiro pelo fator espacial, a localização da Floresta de Galeria, em baixa altitude, não está exposta a fatores meteorológicos (por exemplo, radiação solar) como a Floresta Mesofítica, e segundo pela proximidade do curso d'água. Esse fator espacial e a menor influência dos fatores meteorológicos mantiveram a produção de serapilheira total com um menor desvio médio na Floresta de Galeria em uma escala temporal, sendo que a Floresta Mesofítica ficou susceptível a variações sazonais meteorológicas, tendo assim um maior desvio médio da produção de serapilheira, ao longo do período estudado.
\end{abstract}

Palavras-chave: Fenologia, índice de área foliar, abertura de dossel, pluviosidade.

\section{LITTERFALL PRODUCTION IN GALLERY AND MESOPHYTIC FOREST, IN GARAPA DOLINE, FEDERAL DISTRICT, BRAZIL}

\begin{abstract}
The accumulated litterfall on the soil of forests has an important role in the dynamics of these ecosystems, because the high portion of energy flowing in the system is concentrated in this compartment. The objective this work was to quantify and analyze the litterfall got understory of the Gallery and Mesophytic Forests present in the Dolina da Garapa, and to relate this data with meteorological and ecophysiological data, with aim to check different significance between these two forest environments and between the months of study. From January 2006 to December 2007 were established 50 litterfall trap in each forest environment, where was collected, weighted and classified the litterfall in leaves, stem and trunk, flowers and fruits and seed. The mean values sampled of litterfall production in total mass, leaf, flowers, stem and trunk, fruit and seeds were significatively different in two studied environments. In the correlation carried out between the meteorological and ecophysiological variables and the registered value of litterfall was observed that just the temperature variable had not significance in the coefficient of correlation $(\rho<0.5)$ to litterfall yield. The total production of litterfall during the studied period showed that the Mesophytic Forest is most vulnerable to external meteorological factors and ecophysiological factors of their species, than Gallery Forest. First because the spatial factor, the Forest Gallery location are in low altitude, not being exposed to meteorological factors (e.g., solar radiation) as the Mesophytic,Forest, and second by proximity to the watercourse. This spatial factor and the low influence of meteorological factors kept the total litterfall production with a lower average deviation in the Gallery Forest at a temporal scale, and the Mesophytic Forest was susceptible to seasonal weather, thus having a largest average deviation of the litterfall production a long studied period.
\end{abstract}

Key words: Phenology, leaf area index, open canopy, pluviosity.

${ }^{1}$ Biólogo, Doutor, Bolsista de Pós-Doutorado Júnior do Conselho Nacional de Desenvolvimento Científico e Tecnológico/CNPq Laboratório de Sistemas de Informações Espaciais/LSIE - Departamento de Geografia - Universidade de Brasília/UnB - Rua 16-A, 841, Setor Aeroporto - 74075-150 - Goiânia, GO - otaciliosantana@ gmail.com

${ }^{2}$ Engenheiro Florestal, Ph.D em Inventário e Biometria Florestal, Professor Titular do Departamento de Engenharia Florestal Universidade de Brasília/UnB - Cx. P. 04.357 - 70919-900 - Brasília, DF - imana@unb.br

${ }^{3}$ Biólogo, Mestre em Ecologia, Analista Ambiental do Instituto Brasileiro do Meio Ambiente e Recursos Naturais Renováveis/IBAMA Superintendência do Estado do Tocantins - QD. 102 Sul Cj.01 Lt.05 Av. Joaquim Teotônio Segurado - 77.020-002 - Palmas, TO flaveira@yahoo.com

${ }^{4}$ Engenheiro Florestal, Mestrando em Ciências Florestais - Departamento de Engenharia Florestal - Universidade de Brasília/UnB - Cx. P. 04.357 - 70919-900 - Brasília, DF - gustavo.s.ribeiro@ hotmail.com

Cerne, Lavras, v. 16, n. 4, p. 585-596, out./dez. 2010 


\section{INTRODUÇÃO}

A serapilheira acumulada sobre o solo das florestas tem papel importante na dinâmica desses ecossistemas, pois a maior parcela da energia que flui no sistema está concentrada nesse compartimento, predominando as cadeias tróficas de detritos e as transformações associadas à ciclagem dos nutrientes (DUBBIN et al., 2006), além de proteger o solo de forças erosivas, como chuvas (MORAES et al., 1999). A serapilheira é composta de materiais vegetais depositados na superfície do solo, tais como folhas, cascas, ramos, troncos, gravetos, flores, inflorescências, frutos, sementes e outros fragmentos vegetais (CALDEIRA, 2003; CALDEIRA et al., 2008; VIDAL et al., 2007).

Padrões de produção e acúmulo da serapilheira introduzem também heterogeneidade temporal e espacial no ambiente, podendo afetar a estrutura e a dinâmica da comunidade de plantas, bem como diversos processos do ecossistema (LAURANCE et al., 2002). Portanto, entender os padrões de produção da serapilheira é fundamental para a compreensão da dinâmica e do funcionamento dos ecossistemas, bem como para seu monitoramento (VIDAL et al., 2007). Diversos são os fatores que podem influenciar a sua produção, principalmente: clima, estrutura da vegetação, estádio sucessional da floresta, microambientes, perturbações antropogênicas e adaptações ecofisiológicas (ZALAMEA \& GONZALEZ, 2008).

Os ambientes cársticos, no caso as dolinas, têm sua formação em relevo elaborado pelo processo de corrosão química e pelos processos de abatimento físico, devido principalmente à dissolução da rocha calcária por águas carregadas de ácidos carbônico e húmicos, provenientes da matéria orgânica do solo (GUERRA \& CUNHA, 2007). Pode-se observar o acumulação de detritos ocasionados pelo escorregamento, reforçado por alto declive $\left(>60^{\circ}\right)$, uma área com solo fértil, geralmente cortado por um curso d'água, com o estabelecimento de um ambiente florestal (TATAR et al., 2004). Nesses ambientes, o microclima formado pela geomorfologia somado às condições edáficas do Cerrado, podem ser os fatores decisivos para produção de serapilheira (ACOSTA et al., 2008).

Objetivou-se, neste estudo, quantificar e analisar a serapilheira formada abaixo do dossel da Floresta de Galeria e Mesofítica, na Dolina da Garapa-DF relacionando-a com variáveis meteorológicas e ecofisiológicas, buscando verificar diferenças significativas entre esses dois ambientes florestais e entre os meses de estudo, contribuindo para o conhecimento sobre o Bioma Cerrado.

\section{MATERIAL E MÉTODOS}

A Dolina da Garapa localiza-se a noroeste do Distrito Federal, dentro da Área de Proteção Ambiental da Cafuringa, na cidade satélite de Brazlândia, nas coordenadas geográficas $15^{\circ} 30^{\prime} 19^{\prime \prime} \mathrm{S}$ e $48^{\circ} 06^{\prime} 18^{\prime \prime} \mathrm{W}$, possuindo uma vertente do ribeirão Dois Irmãos, e uma gruta que drena suas águas para o interior (Figura 1).

A margem deste ribeirão, na parte interna da Dolina, a uma altitude de aproximadamente $900 \mathrm{~m}$, situa-se uma Floresta de Galeria que se estabeleceu de forma contínua, sob gleissolos (REATTO et al., 2004). Em levantamento preliminar, as cinco espécies arbóreas ( $>5 \mathrm{~cm}$ de diâmetro) com maiores densidades absolutas (> 50 indivíduos/ha), nesse ambiente florestal, foram: Alibertia macrophylla Schum. (Rubiaceae), Diospyros hispida A. DC. (Ebenaceae), Matayba guianensis Aublet. (Sapindaceae), Pera glabrata Poepp. ex Baill. (Euphorbiacea) e Tapura amazonica Poepp. \& Endl. (Dichapetalace).

Na parte externa da Dolina, na Floresta Mesofítica Decidual ocorre afloramento de calcário, com a classificação do solo de NEOSSOLO LITÓLICO (REATTO et al., 2004) a uma altitude de aproximadamente $1.150 \mathrm{~m}$. As cinco espécies arbóreas (> $5 \mathrm{~cm}$ de diâmetro) com maiores densidades absolutas (> 50 indivíduos/ha), nesse ambiente, foram: Aspidosperma subincanum Mart. (Apocynaceae), Astronium fraxinifolium Schott ex Spreng. (Anacardiaceae), Commiphora leptophloeos (Mart.) J.B.Gillet (Burseraceae), Lonchocarpus montanus Tozzi (Fabaceae) e Sterculia striata A. St.-Hil \& Naud. (Sterculiaceae).

O clima é caracterizado de Aw, segundo a classificação de Köppen (PEEL et al., 2007), apresentando uma temperatura média anual de $21,2^{\circ} \mathrm{C}$, com precipitação em torno de $1.552 \mathrm{~mm} / \mathrm{ano}$ (SECRETRIA DE MEIO AMBIENTE E RECURSOS HÍDRICOS - SEMARH, 2006).

As variáveis meteorológicas obtidas foram a pluviosidade, a temperatura, a umidade e a radiação solar, no período também de janeiro de 2006 a dezembro de 2007. Os dados de pluviosidade foram registrados pela Estação Automática (Brasília, DF) do Instituto Nacional de Meteorologia - INMET (2008), distante $2 \mathrm{~km}$ da área de estudo. A radiação solar foi mensurada por um piranômetro (LI 200SA Pyranometer Sensor). As medidas de temperatura e umidade do ar foram rea-lizadas por um psicrômetro (HMP 35, Vaisala, Inc., Helsinki, Finland). Os equipamentos de medição de radiação, temperatura e umidade foram fixados a 1,5 m do solo, acima de cada coletor instalado. Os dados

Cerne, Lavras, v. 16, n. 4, p. 585-596, out./dez. 2010 


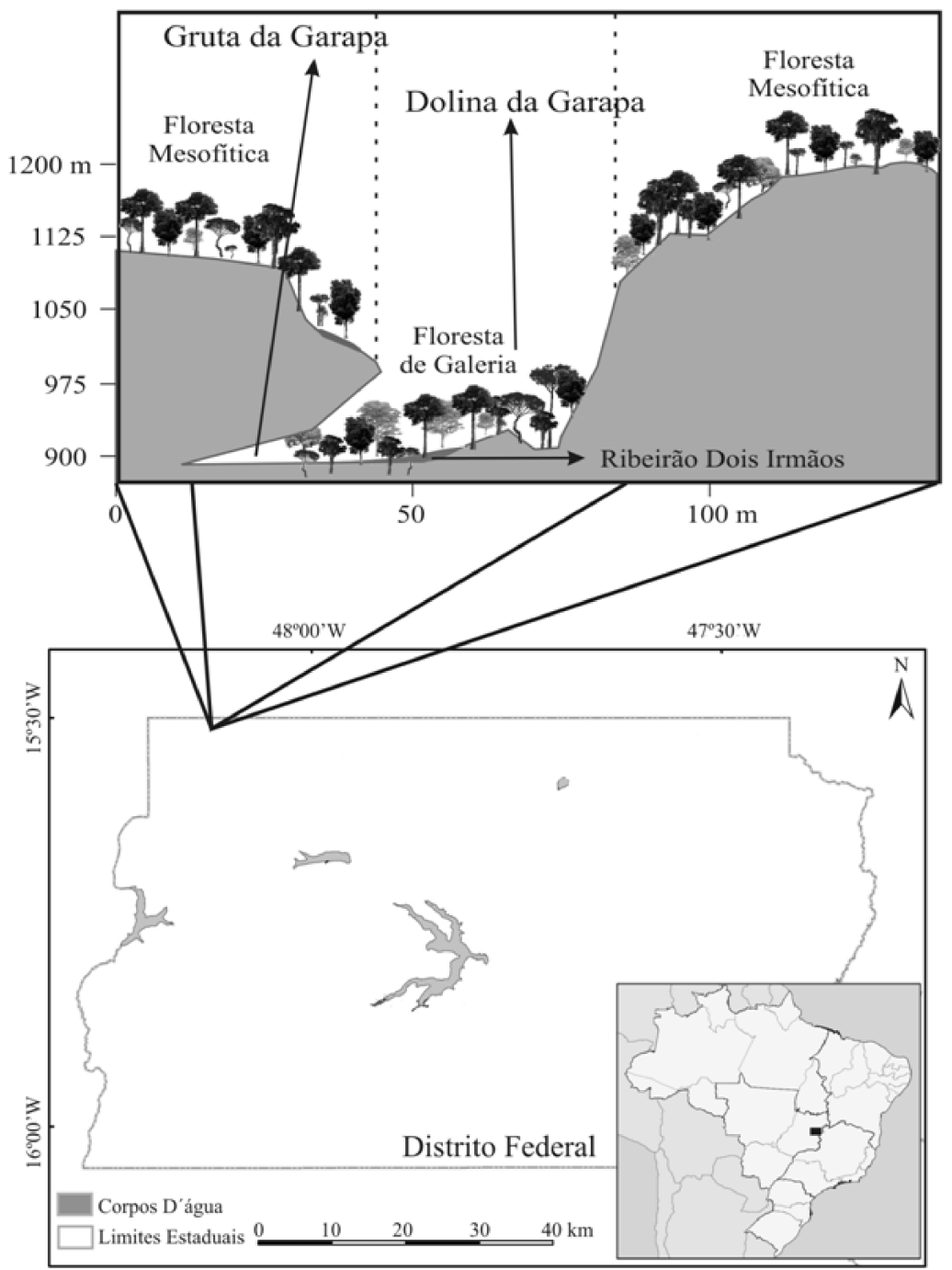

Figura 1 - Localização da área de estudo e esquema de distribuição da Floresta Mesofítica e de Galeria na Dolina da Garapa, Distrito Federal.

Figure 1 - Localization of study area and distribution of Mesophytic and Gallery Forest in the Garapa Doline, Federal District.

foram armazenados por um sistema de aquisição de dados, um Datalog-ger (CR10 X, Campbell Scientific, Inc., UT, USA), programado para fazer leituras das medidas a cada 30 segundos, e armazenar uma média a cada 24 horas. Calibrações dos equipamentos foram realizados segundo Vilani et al. (2006).

As variáveis ecofisiológicas amostradas, também no período de janeiro de 2006 a dezembro de 2007, foram: o índice de área foliar do dossel da floresta (IAF) e a abertura de dossel (\%), quantificados através de um analisador de dossel LAI-2000 (LiCor, Plant Canopy Analyzer). Os dados foram coletados também acima dos coletores $(1,5 \mathrm{~m}$ do solo), ao meio dia, durante 15 dias por mês, havendo dez medidas acima de cada coletor, e com isso estabeleceramse médias mensais.

No interior das florestas foram instalados 100 coletores de serapilheira de $1 \mathrm{~m}^{2}$, feitos de madeira e tela de opacidade de luz para viveiros com furos de $1 \mathrm{~mm}$ de diâmetro para coleta de folhas, flores, pedaço de caule e galhos, frutos e sementes (ZALAMEA \& GONZALEZ,

Cerne, Lavras, v. 16, n. 4, p. 585-596, out./dez. 2010 
2008). Em cada ambiente florestal estudado, a Floresta Galeria e Mesofítica, foram distribuídos 50 coletores, dispostos a $30 \mathrm{~cm}$ do solo, com espaçamentos entre eles de $3 \times 3 \mathrm{~m}$, ocupando uma área total de $324 \mathrm{~m}^{2}$.

As coletas foram realizadas de janeiro de 2006 a dezembro de 2007, no último dia de cada mês, sendo que no primeiro dia do mês seguinte, os coletores eram esvaziados. O material depositado nos coletores foi seco a $60^{\circ} \mathrm{C}$ em estufa de circulação forçada, até peso constante e separados em quatro grupos: a) folhas, b) caules e galhos, c) flores e d) frutos e sementes. Todos foram pesados em balanças eletrônicas de precisão $(0,01 \mathrm{~g})$, e transformados na unidade: $\mathrm{kg} \mathrm{ha}^{-1}$.

Teste estatístico não paramétrico Mann-Whitney foi efetuado para se obter a diferença significativa, através do nível de significância (valor de $\mathrm{p}<0,05$ ), entre as variáveis meteorológicas e ecofisiológicas da Floresta Mesofítica e de Galeria. Para se avaliar o grau de relação entre os valores médios mensais dos coletores da massa de vegetação e das variáveis meteorológicas - pluviosidade, temperatura, umidade e radiação, e das variáveis ecofisiológicas: IAF e abertura de dossel - efetuou-se a correlação não paramétrica de Spearman, para se obter o valor do coeficiente $\rho_{\text {e analisar }}$ possíveis correlações significativas desse coeficiente (- 0,5 $\langle\rho>0,5$ ), isso sem fazer suposições sobre a distribuição de frequências das variáveis (SOKAL \& ROHLF, 1981). Para simplificar interpretações de significância, o coeficiente de Spearman $(\rho)$ foi trabalhado em módulo, não havendo correlações negativas, sendo realizada posteriormente a análise de regressão para ajustar e observar tendências inversas ou diretamente proporcionais, segundo descrito por Sokal \& Rohlf (1981).

Análises de regressão lineares e não lineares foram efetuadas entre os valores médios mensais dos coletores da massa de vegetação e das variáveis meteorológicas: pluviosidade, temperatura, umidade e radiação, e das variáveis ecofisiológicas: IAF e abertura de dossel, para o cálculo do coeficiente de determinação $\left(\mathrm{R}^{2}\right)$, nível de significância do ajuste (valor de p), erro e equação do ajuste. Com a análise de regressão obteve-se, através do ajuste dos valores mensais médios das variáveis amostradas, a forma de distribuição dos dados relacionados, ou seja, a proporcionalidade de distribuição diretamente ou inversamente a partir do acréscimo ou redução do valor da variável independente, no caso as variáveis meteorológicas e ecofisiológicas. Todas as análises, desvios estatísticos e gráficos foram calculados pelo programa Statistica 5.0 (STATSOFT, 2000).

Cerne, Lavras, v. 16, n. 4, p. 585-596, out./dez. 2010

\section{RESULTADOS E DISCUSSÃO}

As variações sazonais observadas no regime das chuvas no período seco (maio a outubro) e no período chuvoso (novembro a abril) (Figura 2) foram também observadas na variável temperatura, umidade e radiação (Figura 3). Nessas, as médias foram significativamente diferentes $(p<0,001)$ no Teste Mann-Whitney, entre os ambientes florestais, significando diferentes microclimas nos ambientes dentro (Floresta de Galeria) e fora (Floresta Mesofítica) da Dolina da Garapa. A temperatura e radiação foram em média maiores na Floresta Mesofítica $19,7 \pm 0,8^{\circ} \mathrm{C}$ e $724 \pm 157 \mathrm{~kJ} \mathrm{~m}^{2}$, do que na Floresta de Galeria $17,1 \pm 0,6^{\circ} \mathrm{C}$ e $428 \pm 52 \mathrm{~kJ} \mathrm{~m}^{2}$, respectivamente. A variável umidade apresentou padrão oposto, em média foi maior na Floresta de Galeria $(83 \pm 12 \%)$ do que na Floresta Mesofítica $(59 \pm 21 \%)$. Segundo Acosta et al. (2008), isso pode ser explicado pela posição geomorfológica na qual os ambientes estão inseridos, obtendo-se diferentes microclimas. Na Floresta de Galeria, a menor incidência solar e de ventos mantém a umidade por estar situada entre declives, além de situada às margens de um curso d'água.

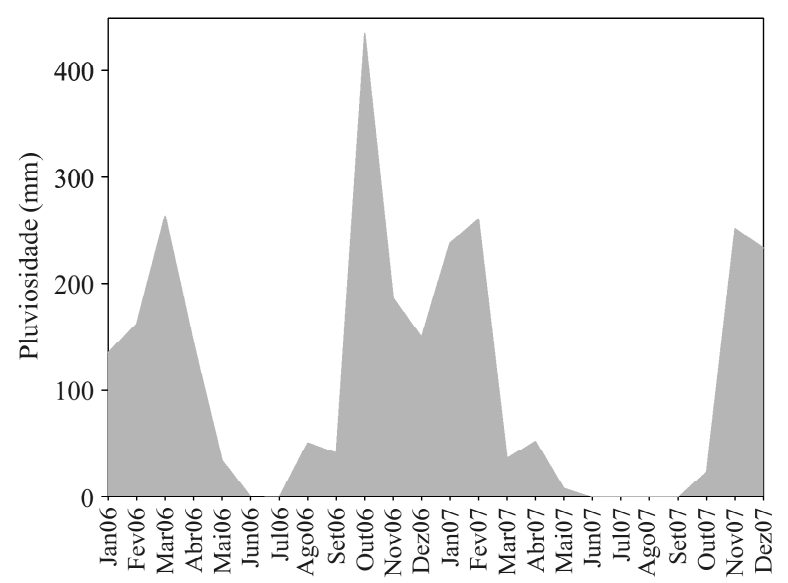

Figura 2 - Pluviosidade registrada no período de estudo, de janeiro de 2006 a dezembro de 2007 (INMET, 2008).

Figure 2 - Sampled pluviosity in studied period, from January, 2006 to December, 2007 (INMET, 2008).

Na Floresta de Galeria, os valores médios de IAF foram maiores $(4,1 \pm 0,4)$ do que para Floresta Mesofítica $(2,0 \pm 0,9)$, e menores para abertura de dossel, $78 \pm 9 \%$ e $37 \pm 4 \%$, respectivamente (Figura 4), no período estudado. Os dados de IAF e abertura de dossel encontrados foram semelhantes aos dados descritos pela literatura, para esse 

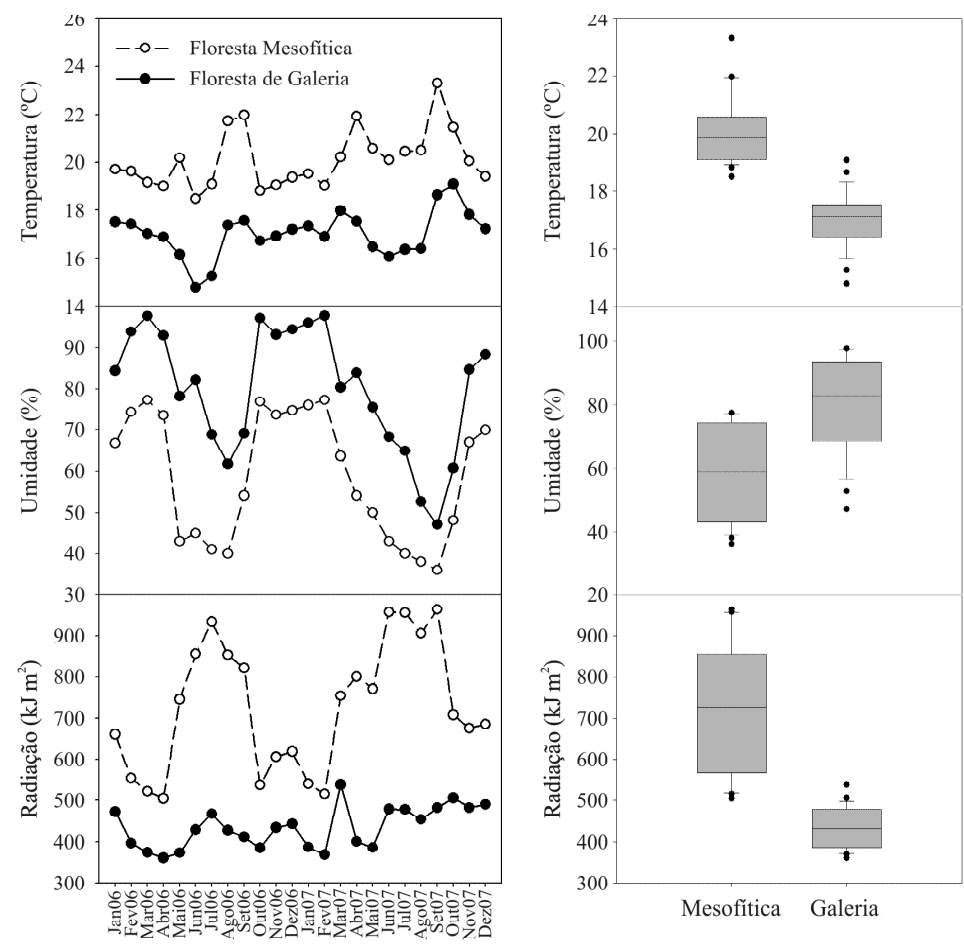

Figura 3 - Distribuição média mensal, nos anos de 2006 e 2007, da temperatura, umidade e radiação dentro da Floresta Mesofítica e de Galeria, na Dolina da Garapa; e desvios da média nos dois ambientes florestais.

Figure 3 - Monthly average distribution, in 2006 and in 2007, of temperature, humidity and radiation in Mesophytic and Gallery Forest, in the Garapa Doline; and average deviation in the two forest environments.
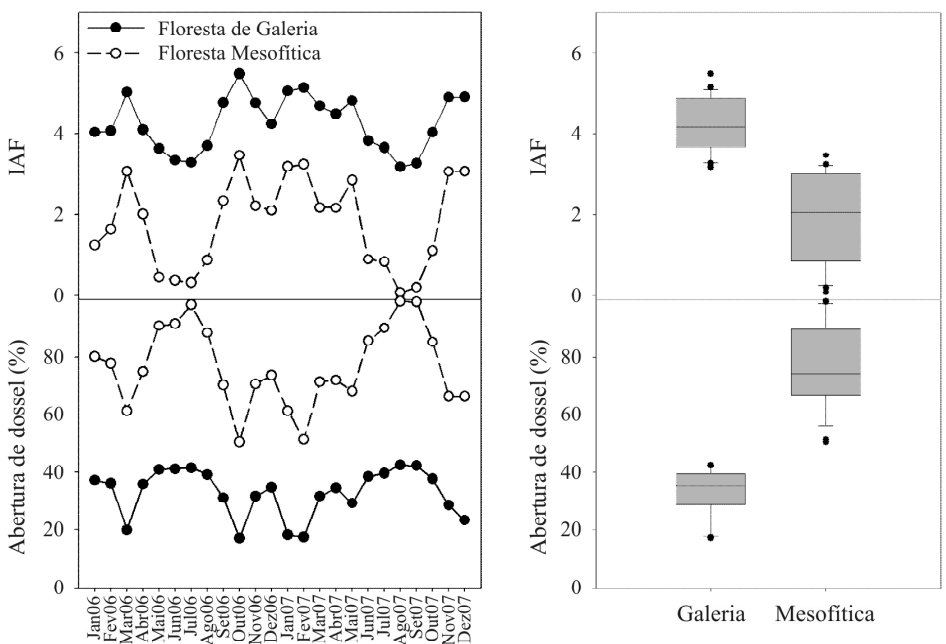

Figura 4 - Distribuição média mensal, nos anos de 2006 e 2007, do índice de área foliar (IAF) e abertura de dossel (\%) da Floresta Mesofítica e de Galeria, na Dolina da Garapa; e desvios da média nos dois ambientes florestais.

Figure 4-Monthly average distribution, in the years of 2006 and 2007, of leaf area index (LAI) and open canopy (\%) in Mesophytic and Gallery Forest, in the Garapa Doline; and average deviation in the two forest environments.

Cerne, Lavras, v. 16, n. 4, p. 585-596, out./dez. 2010 
ambiente, 2,5 e $65 \%$, adotando-se a mesma metodologia (HOFFMANN et al., 2004; SIMONI et al., 2003). Diferenças significativas ( $\mathrm{p}<0,0125$ ), no Teste Mann-Whitney, foram observadas, nos valores de IAF e abertura de dossel (AD), entre a Floresta de Galeria e a Floresta Mesofítica. Caylor et al. (2005) e Rodriguez-Calcerrada et al. (2008) observaram esses comportamentos sazonais das variáves IAF e abertura de dossel, em florestas savânicas, como respostas ecofisiológicas dos indivíduos vegetais, que regulam a perda hídrica pelos estômatos, reduzindo a área de sua parte aérea e todo o processo fotossintético e de evapotranspiração vegetal, em períodos secos.

Essa regulação e perda de área foliar refletiram-se na produção de massa vegetal de serapilheira, conforme observase nas Figuras 5 e 6, indicando também uma variação sazonal dessa produção nos coletores de todos os componentes da biomassa, principalmente para os registros na Floresta Mesofítica. A Floresta Mesofítica, nos períodos chuvosos produziram, em média, até seis vezes mais serapilheira total $\left(750 \pm 127 \mathrm{~kg} \mathrm{ha}^{-1}\right)$, do que nos períodos secos $\left(123 \pm 34 \mathrm{~kg} \mathrm{ha}^{-1}\right)$, tendo maior variação no grupo formado por folhas $( \pm 150$ $\mathrm{kg} \mathrm{ha}^{-1}$ ), sendo as maiores perdas de material vegetal por indivíduo, conforme observa-se na Figura 5 e está corroborado por Nascimento et al. (2007). A sazonalidade também foi confirmada pelos desvios estatísticos (Figura 6), mostrando a diferença dos maiores para os menores outliers, o que implica na diferença de massa coletada, durante os períodos pluviométricos existentes, já que os valores de massa $\left(\mathrm{kg} \mathrm{ha}^{-1}\right)$ obtiveram diferença significativas $(\mathrm{p}<0,001)$ apenas entre os ambientes florestais no componente frutos e

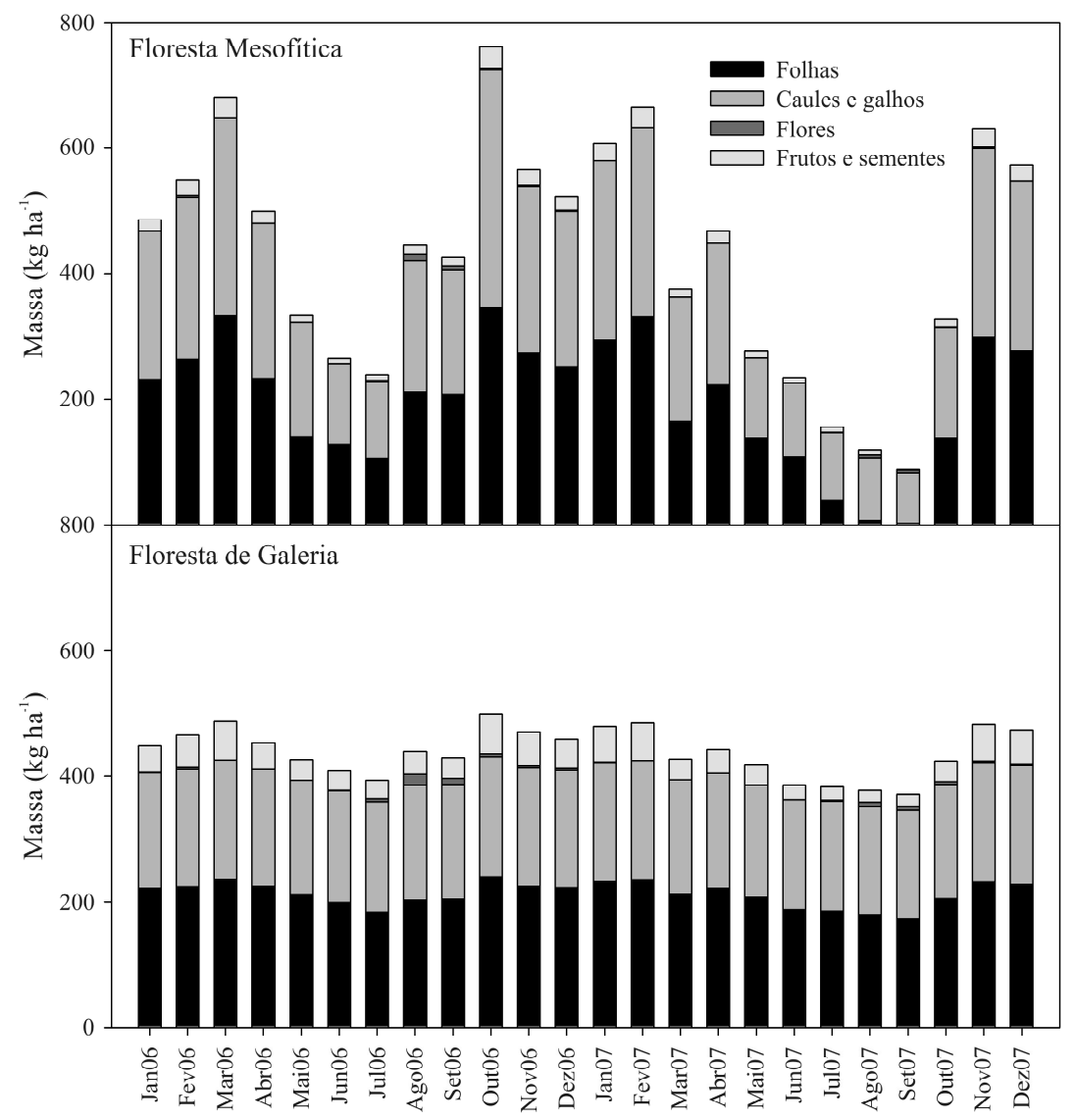

Figura 5 - Distribuição da massa de serapilheira coletada $\left(\mathrm{kg} \mathrm{ha}^{-1}\right)$ no período de estudo, classificada em quatro grupos: a) folhas, b) caules e galhos, c) flores, d) frutos e sementes.

Figure 5 - Mass distribution of sampled litterfall $\left(\mathrm{kg} \mathrm{ha}^{-1}\right)$ in studied period, classified in four groups: a) leaves, b) trunk and branches, c) flowers and d) fruits and seeds.

Cerne, Lavras, v. 16, n. 4, p. 585-596, out./dez. 2010 

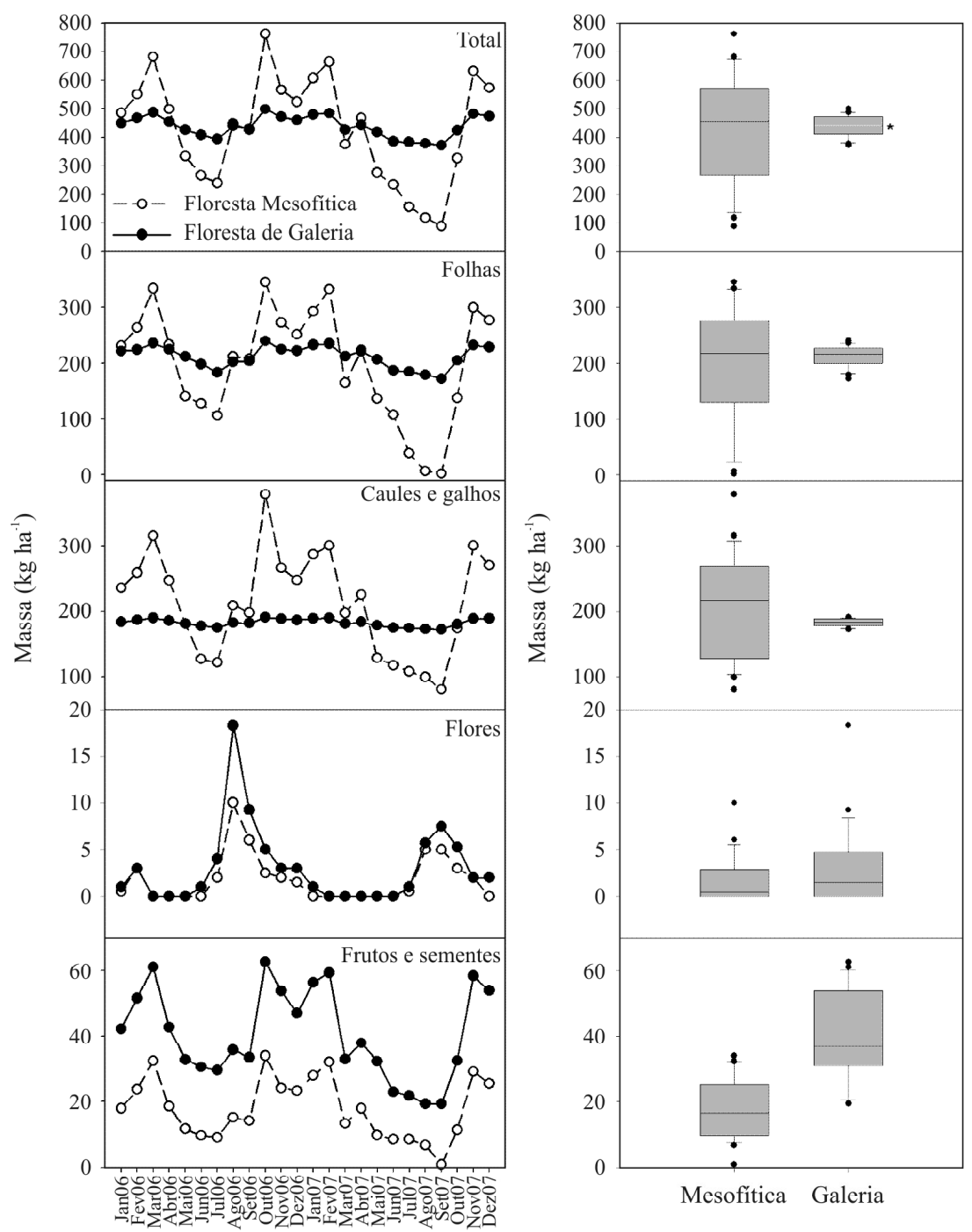

Figura 6 - Distribuição média mensal, nos anos de 2006 e 2007, da massa total, de folhas, caules e galhos, flores e frutos e sementes registrados abaixo do dossel da Floresta Mesofítica e de Galeria, na Dolina da Garapa-DF; e desvios da média nos dois ambientes florestais.

Figure 6-Monthly average distribution, in the years of 2006 and 2007, of total, leaves, trunk and branches, flowers and fruits and seeds mass, lower canopy of Mesophytic and Gallery Forest, in Garapa Doline-DF; and average deviation in the two forest environments.

sementes, $18 \pm 5 \mathrm{~kg} \mathrm{ha}^{-1}$ para Floresta de Galeria e $37 \pm 12 \mathrm{~kg} \mathrm{ha}^{-1}$ para Floresta Mesofítica, encontrada pelo Teste MannWhitney, entre os meses estudados.

Os valores médios totais de massa encontradas nos coletores entre os ambientes florestais foram de $446 \pm 172$ $\mathrm{kg} \mathrm{ha}^{-1}$, na Floresta de Galeria, e $451 \pm 31 \mathrm{~kg} \mathrm{ha}^{-1}$ na Floresta Mesofítica (Figura 6). Esses valores refletiram as diferenças observadas nos valores de IAF e abertura de dossel, durante os meses estudados. Os valores encontrados de massa foram baixos em relação aos dados de formação de serapilheira em outros ambientes florestais brasileiros. $\mathrm{Na}$ Floresta Atlântica, foram mensurados $965 \mathrm{~kg} \mathrm{ha}^{-1}$ (VIBRANS \& SEVEGNANI, 2000); em Cerradão (CIANCIARUSO et al., 2006), $780 \mathrm{~kg} \mathrm{ha}^{-1}$; na Floresta

Cerne, Lavras, v. 16, n. 4, p. 585-596, out./dez. 2010 
Mesófila Semidecídua (MARTINS \& RODRIGUES, 1999) $596 \mathrm{~kg} \mathrm{ha}^{-1}$; e na Floresta Amazônica (DANTAS \& PHILLIPSON, 1989) $504 \mathrm{~kg} \mathrm{ha}^{-1}$. Segundo Dublin et al. (2006), em solos calcários, com rochas expostas e solos rasos, há menos indivíduos arbóreos em relação a outros ambientes florestais, pela carência do substrato para vegetação, o que pode justificar essa menor produção de serapilheira.

Na Floresta Mesofítica estudada, a maior produção de serapilheira total ao longo do ano foi principalmente no início (março) e final do período seco (agosto e setembro), devido ao início da seca pela perda de folhas dos indivíduos arbóreos para adaptações ecofisiológicas de regulação hídrica, durante o período limitante de água (FRANCO, 2002), e no final pela produção emergente de folhas e pela produção de flores, pouco acentuada em outros meses do ano. Isso não foi observado acentuadamente na Floresta de Galeria, apenas a produção de frutos e sementes, mais dependentes de fatores ecofisiológicos das espécies do que a fatores externos (OLIVEIRA \& GIBBS, 2002).

Realizando a correlação entre as variáveis meteorológicas e ecofisiológias e o valor de serapilheira encontrada (Tabela 1), observou-se que, apenas a variável temperatura não obteve significância do coeficiente de correlação $(\rho<0,5)$, para produção de serapilheira. Nas variáveis com coeficientes de correlação significativos ( $\rho>0,5$ ), os valores de $\rho$ foram maiores em média para a Floresta Mesofítica $(\rho=0,817 \pm 0,121)$ do que para Floresta de Galeria $(\rho=0,613 \pm 0,219)$ na produção total de serapilheira, comprovando-se que a Floresta Mesofítica é a mais vulnerável a fatores externos meteorológicos e a fatores ecofisiológicos de suas espécies do que a Floresta de Galeria, corroborando com Sampaio et al. (2007). A variável pluviosidade, umidade, abertura de dossel e IAF seguiram essa tendência de correlação maior para Floresta Mesofítica em todos os grupos vegetais de serapilheira estabelecidos, folhas, caules e galhos, flores, frutos e sementes, sendo que apenas a correlação entre os valores de umidade e a produção de serapilheira de flores da Floresta de Galeria não obtiveram coeficiente de correlação significativo. Já a correlação de produção de serapilheira com a variável radiação obteve o coeficiente de correlação significativo $(\rho<0,711)$ apenas para Floresta Mesofítica, pois a Floresta de Galeria não possui períodos acentuados de caducifolia como a Floresta Mesofítica, e os indivíduos arbóreos mantiveram suas folhas praticamente o ano inteiro, o que não ocorre com a Floresta Mesofítica em que houve uma queda na produção de serapilheira de folhas e de índice de área foliar, e com isso um aumento do valor médio da radiação no interior desse ambiente florestal, conforme já observou-se nas Figuras 5 e 6, e também na literatura (MORAES et al., 1999; NASCIMENTO et al., 2007).

A análise de regressão realizada entre os valores em massa de serapilheira com as variáveis meteorológicas e ecofisiológicas (Figura 7 e Tabela 2) permitiu observar três comportamentos de relação com a produção de serapilheira: a) diretamente proporcional, com

Tabela 1 - Correlação de Spearman entre as variáveis meteorológicas e ecofisiológicas e a massa de serapilheira nos ambientes florestais estudados, Floresta Mesofítica $=\mathrm{M}$ e Floresta de Galeria = G.

Table 1 - Spearman correlation between meteorological and ecophysiological variables and litterfall mass in studied forest environments, Mesophytic Forest $=M$ and Gallery Forest $=G$.

\begin{tabular}{|c|c|c|c|c|c|c|c|c|c|c|}
\hline \multirow{3}{*}{ Relação } & \multicolumn{2}{|c|}{ Folhas } & \multirow{2}{*}{\multicolumn{2}{|c|}{ Caules e galhos }} & \multirow{2}{*}{\multicolumn{2}{|c|}{$\begin{array}{l}\text { Flores } \\
\qquad \mathrm{kg} \mathrm{ha}^{-1}\end{array}$}} & \multicolumn{2}{|c|}{ Frutos e sementes } & \multicolumn{2}{|c|}{ Total } \\
\hline & & & & & & & & & & \\
\hline & M & G & M & $\mathrm{G}$ & M & G & M & G & M & G \\
\hline Pluviosidade (mm) & $0,905^{*}$ & $0,783 *$ & $0,946 *$ & $0,676^{*}$ & $0,907 *$ & $0,586 *$ & $0,951^{*}$ & $0,811^{*}$ & $0,927 *$ & $0,714^{*}$ \\
\hline Temperatura $\left({ }^{\circ} \mathrm{C}\right)$ & 0,400 & 0,294 & 0,247 & 0,131 & 0,330 & 0,150 & 0,344 & 0,372 & 0,331 & 0,237 \\
\hline Umidade (\%) & $0,912 *$ & $0,599 *$ & $0,762 *$ & $0,611^{*}$ & $0,701 *$ & 0,411 & $0,804^{*}$ & $0,652 *$ & $0,795 *$ & $0,568^{*}$ \\
\hline Radiação (kJ m²) & $0,765^{*}$ & 0,142 & $0,834 *$ & 0,422 & $0,782 *$ & 0,243 & $0,711^{*}$ & 0,287 & $0,773 *$ & 0,274 \\
\hline Abertura de dossel (\%) & $0,848^{*}$ & $0,776^{*}$ & $0,813^{*}$ & $0,736^{*}$ & $0,828 *$ & $0,767 *$ & $0,711^{*}$ & $0,714 *$ & $0,800 *$ & $0,748^{*}$ \\
\hline IAF & $0,792 *$ & $0,753 *$ & $0,738 *$ & $0,703 *$ & $0,793 *$ & $0,788 *$ & $0,832 *$ & $0,792 *$ & $0,789 *$ & $0,759^{*}$ \\
\hline
\end{tabular}

* Correlação significativa $\rho>0,5$

* Significance of correlation $\rho>0.5$

Cerne, Lavras, v. 16, n. 4, p. 585-596, out./dez. 2010 

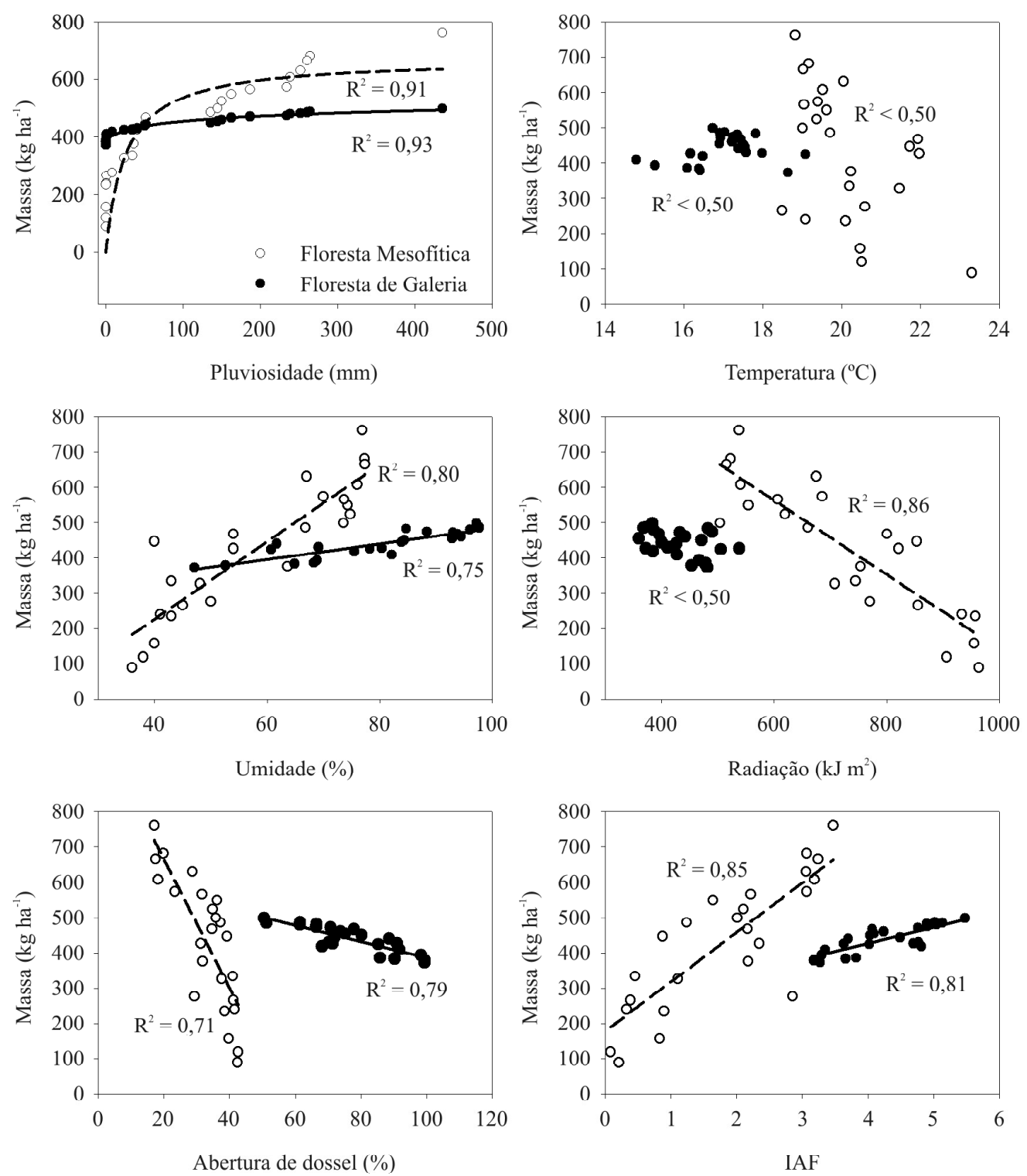

Figura 7 - Ajuste da análise de regressão entre a massa total (somatório de folhas, flores, caules, galhos, frutos e sementes), coletada abaixo do dossel da Floresta Mesofítica e de Galeria, na Dolina da Garapa, com as variáveis: pluviosidade, umidade, temperatura, radiação, abertura de dossel e índice de área foliar (IAF). $\mathrm{R}^{2}=$ coeficiente de determinação do ajuste.

Figure 7 - Adjusts of regression analysis between the total mass (somatory of leaf, flowers, trunk, branches, fruits and seeds) collected lower canopy in the Mesophytic and Gallery Forest, in the Garapa Doline, with the variables: pluviosity, humidity, temperature, radiation, open canopy and leaf area index $(L A I) . R^{2}=$ coefficient of determination of adjusts.

pluviosidade, umidade e IAF; b) inversamente proporcional, com radiação (exceto os dados da Floresta Mesofítica, não significativo) e abertura de dossel; e c) não significativo, com a temperatura. Haase \& Hirooka (1998) e Sampaio et al. (2007) observaram que a disponibilidade de água foi um dos principais fatores para produção de serapilheira, principalmente para Florestas Mesofíticas, que tiveram uma maior variação em sua umidade interna $( \pm 18,49)$ do que na Floresta de Galeria $( \pm 15,23)$. Como pluviosidade e umidade são fatores que

Cerne, Lavras, v. 16, n. 4, p. 585-596, out./dez. 2010 
Tabela 2 - Parâmetros da análise de regressão entre a massa vegetal e as variáveis: pluviosidade, temperatura, umidade, radiação, abertura de dossel e índice de área foliar (IAF).

Table 2 - Parameters of regression analysis between the plant mass and the variables: pluviosity, temperature, humidity, radiation, open canopy and leaf area index (LAI).

\begin{tabular}{|c|c|c|c|c|c|c|}
\hline & Relação & Floresta & Equação* & $\mathrm{R}^{2}$ & $\mathrm{p}$ & Erro \\
\hline \multirow{12}{*}{ 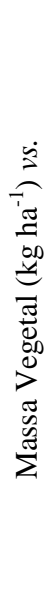 } & \multirow{2}{*}{ Pluviosidade (mm) } & Mesofítica & $\mathrm{y}=2,096+4,780\left(1-\mathrm{e}^{-0,008 x}\right)$ & 0,91 & $<0,001$ & 0,002 \\
\hline & & Galeria & $y=3,921+0,929\left(1-e^{-0,001 x}\right)$ & 0,93 & $<0,001$ & 0,014 \\
\hline & \multirow{2}{*}{ Temperatura $\left({ }^{\circ} \mathrm{C}\right)$} & Mesofítica & - & $<0,50$ & - & - \\
\hline & & Galeria & - & $<0,50$ & - & - \\
\hline & \multirow{2}{*}{ Umidade (\%) } & Mesofítica & $y=2,126+0,109 x$ & 0,80 & 0,002 & 0,068 \\
\hline & & Galeria & $y=2,642+0,021 x$ & 0,75 & 0,001 & 0,011 \\
\hline & \multirow{2}{*}{ Radiação (kJ m²) } & Mesofítica & $y=10,194-0,002 x$ & 0,86 & $<0,001$ & 0,029 \\
\hline & & Galeria & - & $<0,50$ & - & - \\
\hline & \multirow{2}{*}{ Abertura de dossel (\%) } & Mesofítica & $y=10,310-0,182 x$ & 0,71 & $<0,001$ & 0,012 \\
\hline & & Galeria & $y=6,158-0,029 x$ & 0,79 & 0,003 & 0,051 \\
\hline & \multirow{2}{*}{ IAF } & Mesofítica & $y=1,780+1,401 x$ & 0,85 & $<0,001$ & 0,033 \\
\hline & & Galeria & $y=2,442+0,456 x$ & 0,81 & $<0,001$ & 0,009 \\
\hline
\end{tabular}

*Equação, $\mathrm{R}^{2}$ = coeficiente de determinação, $\mathrm{p}$ = nível de significância e Erro do ajuste.

*Equation, $R^{2}=$ coefficient of determination, $p=$ significant level and Adjust Error.

aumentam a disponibilidade de água, juntamente com o aumento dos valores de IAF, a produção em massa de serapilheira foi aumentada, diferentemente de variáveis como a radiação e abertura de dossel, conforme observase na Figura 7. Os dados de temperatura foram não significativos com relação à produção em massa de serapilheira $\left(\mathrm{R}^{2}<0,5\right.$ e $\left.\mathrm{p}>0,05\right)$. Segundo Haase \& Hirooka (1998), isso se deve à pouca variação da temperatura nos trópicos $( \pm 3,19)$, em relação a regiões temperadas $( \pm 15,99)$, e também quando a temperatura aumenta durante o ano, sem pluviosidade e umidade, os indivíduos arbóreos tendem a entrar em senescência, evitando a perda hídrica e cessando a produção de área foliar (FRANCO, 2002).

Os dados deste trabalho corroboraram com os estudos de Rodriguez-Calcerrada et al. (2008), em seus estudos com florestas montanhosas na Espanha, e Zalamea \& Gonzalez (2008), em florestas tropicais de Porto Rico, que verificaram que o regime hídrico é significativamente determinante para a regulação sazonal dos processos ecofisiológicos das espécies florestais, principalmente em florestas distantes do curso d'água, como é o caso da Floresta Mesofítica, onde em alguns casos, ocorre a caducifolia, reduzindo a produção foliar e de material botânico e consequentemente a deposição de serapilheira, diferentemente da Floresta de Galeria

Cerne, Lavras, v. 16, n. 4, p. 585-596, out./dez. 2010

\section{CONCLUSÕES}

A sazonalidade da distribuição da pluviosidade durante o ano influenciou diretamente os índices de área foliar e de abertura de dossel, resultado de adaptações ecofisiológicas das espécies presentes nas áreas de estudo. Por sua vez, isso também influenciou na produção de serapilheira, tendo maior produção no início (março) e final da época seca (agosto e setembro), no início da seca, pela perda de folhas dos indivíduos arbóreos para adaptações ecofisiológicas de regulação hídrica durante o período limitante de água, e no final, pela produção emergente de folhas e pela produção de flores, pouco acentuada em outros meses do ano.

Entre as variáveis meteorológicas e ecofisiológias e o valor de serapilheira registrado, observou-se que apenas a variável temperatura não obteve significância do coeficiente de correlação para produção de serapilheira. Nas variáveis com correlação significativa, os maiores valores dos coeficientes foram obtidos mais na Floresta Mesofítica do que na Floresta de Galeria, na produção total de serapilheira.

Assim, pela produção total de serapilheira, durante o período estudado, concluiu-se que a Floresta Mesofítica é mais vulnerável a fatores externos meteorológicos e a 
fatores ecofisiológicos de suas espécies, do que a Floresta de Galeria. Primeiro, pelo fator espacial, a localização da Floresta de Galeria, em baixa altitude, não está exposta a fatores meteorológicos (por exemplo radiação solar) como a Floresta Mesofítica, e segundo pela proximidade do curso d'água. Esse fator espacial e a menor influência dos fatores meteorológicos mantiveram a produção de serapilheira total com um menor desvio médio na Floresta de Galeria em uma escala temporal, durante o período de estudo, sendo que a Floresta Mesofítica ficou susceptível a variações sazonais meteorológicas, tendo assim um maior desvio médio da produção de serapilheira, ao longo do período estudado.

\section{AGRADECIMENTOS}

Ao CNPq e CAPES, pelo auxílio financeiro. Ao Departamento de Engenharia Florestal da Universidade de Brasília, pela logística.

\section{REFERÊNCIAS BIBLIOGRÁFICAS}

ACOSTA, B,; SANCHEZ-JARDON, L.; DEL POZO, A. Grassland species composition and morpho-functional traits along an altitudinal gradient in a Mediterranean environment: relationship with soil water availability and evaporative dynamic. Acta Oecologica, Paris, v. 34, n. 1, p. 26-37, Jan. 2008.

CALDEIRA, M. V. W. Determinação de biomassa e nutrientes em uma Floresta Ombrófila Mista Montana em General Carneiro, Paraná. 2003. Tese (Doutorado em Ciências Florestais) - Universidade Federal do Paraná, Curitiba, 2003.

CALDEIRA, M. V. W.; VITORINO, M. D.; SCHAADT, S. S.; MORAES, E.; BALBINOT, R. Quantificação de serapilheira e de nutrientes em uma Floresta Ombrófila Densa. Semina: Ciências Agrárias, Londrina, v. 29, n. 1, p. 53-68, jan./mar. 2008.

CAYLOR, K. K.; SHUGART, H. H.; RODRIGUEZ-ITURBE, I. Tree canopy effects on simulated water stress in Southern African Savannas. Ecosystems, New York, v. 8, p. 17-32, Jan. 2005.

CIANCIARUSO, M. V.; PIRES, J. S. R.; DELITTI, W. B. C.; SILVA, É. F. L. P. Litter fall and leaf decomposition in cerradão Jataí Reserve, municipality of Luiz Antônio, São Paulo State, Brazil. Acta Botânica Brasilica, São Paulo v. 20, n. 1, p. 49-59, jan./mar. 2006.
DANTAS, M.; PHILIPSON, J. Litterfall and litter nutrient content in primary and secondary Amazonian "terra firme" rain forest. Journal of Tropical Ecology, Cambridge, v. 5, n. 1, p. 27-36, Feb. 1989.

DUBBIN, W. E.; PENN, M. G.; HODSON, M. E. Edaphic influences on plant community adaptation in the Chiquibul forest of Belize. Geoderma, Tucson, v. 131, n. 1/2, p. 76-88, Mar. 2006.

FRANCO, A. C. Ecophysiology of woody plants. In: PAULO, S.; OLIVEIRA, R. J. M. (Orgs.). The Cerrados of Brazil: ecology and natural history of a neotropical savanna. Washington: Columbia University, 2002. v. 1, p. 329-347.

GUERRA, A. J. T.; CUNHA, S. B. Geomorfologia: uma atualização de bases e conceitos. Rio de Janeiro: Bertrand Brasil, 2007. $472 \mathrm{p}$.

HAASE, R.; HIROOKA, R. Y. Structure, composition and small litter dynamics of a semi-deciduous forest in Mato Grosso, Brazil. Flora, São Paulo, v. 93, n. 2, p. 141-147, jan./ mar. 1998.

HOFFMANN, W. A.; ORTHEN, B.; FRANCO, A. C. Constraints to seedling success of savanna and forest trees across the savanna-forest boundary. Oecologia, Berlin, v. 140, p. 252 260, Fev. 2004.

INSTITUTO NACIONAL DE METEOROLOGIA. Disponivel

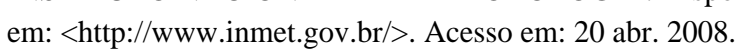

LAURANCE, W. F.; LOVEJOY, T. E.; VASCONCELOS, H. L.; BRUNA, E. M.; DIDHAM, R. K.; STOUFFER, P. C.; GASCON, C.; BIERREGAARD, R. O.; LAURANCE, S. G.; SAMPAIO, E. Ecosystem decay of Amazonian forest fragments: a 22-year investigation. Conservation Biology, Hoboken, v. 16, p. 605-618, Mar. 2002.

MARTINS, S. V.; RODRIGUES, R. R. Produção de serapilheira em clareiras de uma floresta estacional semidecidual no município de Campinas, SP. Revista Brasileira Botânica, São Paulo, v. 22, n. 3, p. 405-412, dez. 1999.

MORAES, R. M.; DELITTI, W. B. C.; VUONO, Y. S. Litterfall and litter nutrient content in two Brazilian Tropical Forests. Revista Brasileira de Botânica, São Paulo, v. 22, p. 9-16, abr. 1999.

Cerne, Lavras, v. 16, n. 4, p. 585-596, out./dez. 2010 
NASCIMENTO, A. R. T.; FELFILI, J. M.; FAGG, C. W. Canopy opennes and LAI estimates in two seasonally deciduous forests on limestone outcrops in central Brazil using hemispherical photographs. Revista Árvore, Viçosa, v. 31, n. 1, p. 167-176, jan./mar. 2007.

OLIVEIRA, P. E. A. M.; GIBBS, P. E. Pollination and reproductive biology in cerrado plant communities. In: PAULO, S.; OLIVEIRA, R. J. M. (Orgs.). The Cerrados of Brazil: ecology and natural history of a neotropical savanna. Washington: Columbia University, 2002. v. 1, p. 329-347.

PEEL, M. C.; FINLAYSON, B. L.; MCMAHON, T. A. Updated world map of the Köppen-Geiger climate classification. Hydrological and Earth System Science, Delft, v. 11, n. 10, p. 1633-1644, Oct. 2007.

REATTO, A.; MARTINS, E. S.; FARIAS, M. R.; SILVA, A. V.; CARVALHO JUNIOR, O. A. Mapa pedológico digital SIG atualizado do Distrito Federal: escala 1:100.000 e uma síntese do texto explicativo. Planaltina: Embrapa Cerrados, 2004. 31 p (Documentos, 120).

RODRIGUEZ-CALCERRADA, J.; MUTKE, S.; ALONSO, J. Influence of overstory density on understory light, soil moisture, and survival of two underplanted oak species in a Mediterranean montane Scots pine forest. Investigacion Agraria-Sistemas y Recursos Forestales, Madrid, v. 17, n. 1, p. 31-38, Jan. 2008.

SAMPAIO, A. B.; HOLL, K. D.; SCARIOT, A. Does restoration enhance regeneration of seasonal deciduous forests in pastures in central Brazil? Restorarion Ecology, Tucson, v. 15, n. 3, p. 462471, July 2007.
SECRETRIA DE MEIO AMBIENTE E RECURSOS HÍDRICOS. APA de Cafuringa, a última fronteira natural do DF. Brasília, 2006. 544 p.

SOKAL, R. R.; ROHLF, F. J. Biometry. New York: Freeman, 1981. 429 p.

STATSOFT. Statistica for Windows: computer program manual. Tulsa, 2000. 323 p.

TATAR, E.; MIHUCZ, V. G.; TOMPA, K. Study of soil leachates in doline above the Beke Cave, Hungary. Geoderma, Amsdertam, v. 120, n. 1/2, p. 155-164, Jan./Feb. 2004.

VIBRANS, A. C.; SEVEGNANI, L. Produção de serapilheira em dois remanescentes da floresta ombrófila densa em Blumenau, SC. Revista de Estudos Ambientais, Blumenau, v. 1, n. 2, p. 103-116, jan. 2000.

VIDAL, M. M.; PIVELLO, V. R.; MEIRELLES, S. T.; METZGER, J. P. Produção de serapilheira em floresta Atlântica secundária numa paisagem fragmentada (Ibiúna, SP): importância da borda e tamanho dos fragmentos. Revista Brasileira de Botânica, São Paulo, v. 30, n. 3, p. 521-532, jul./set. 2007.

VILANI, M. T.; SANCHES, L.; NOGUEIRA, J. S.; PRIANTE FILHO, N. Sazonalidade da radiação, temperatura e umidade em uma floresta de transição Amazônia Cerrado. Revista Brasileira de Meteorologia, São José dos Campos, v. 21, n. 3b, p. 119 131, jul./set. 2006.

ZALAMEA, M.; GONZALEZ, G. Leaffall phenology in a subtropical wet forest in Puerto Rico: from species to community patterns. Biotropica, Washington, v. 40, n. 3, p. 295-304, Mar. 2008. 